\title{
A New Strategy for Representation and Control of Self-Contained Power Systems
}

\author{
Kent Davey, Fellow, IEEE and Robert Hebner, Fellow, IEEE
}

\begin{abstract}
Power systems on naval vessels and airplanes are good examples of self contained power systems. State of the art LEM modules and voltage sensors provide real time current and voltage data. This paper shows how that information can be used to construct dynamic equivalent impedance representations of the system discretized into key trunk lines. Error analysis indicates that the algorithm performing this representation can be updated in one fifth of a cycle if the signal to noise data ratio is $5 \%$ or less. The magnitude of the equivalent impedance is sufficient to determine when fault control on either end of the trunk line is required. These same equivalent impedances can be used to determine the best switch configuration to maximize load power while minimizing transmission loss subject to line load capability. The accuracy of the equivalent impedance approach is assessed for transients with active and passive loads.
\end{abstract}

Key Words - reconfiguration, phase analysis, transient, fault detection

\section{INTRODUCTION}

Load flow and state analysis characterize the state of the art for modeling and control of power systems. The system equations are typically solved in phasor mode or in some cases in real time if transient information is required. Differential relays remain the primary device for managing faults. The approach adopted in this paper is to break the system into a grid of primary sections or trunk lines, with each section represented by two equal series impedances, and a parallel impedance. These impedances are dynamic and are updated using the current and voltage on both ends of the line. A fault is indicated by a drop in the parallel impedance magnitude.

Phasor estimates of noisy time harmonic signals have been proposed with a variety of models. Pradham [1] proposes a Kalman filter for this task, but the technique requires one cycle to get useful information. Kamwa [2] uses a recursive least squares approach to estimate phase and frequency supplemented by forgetting and adaptive factors. The system is reasonably fast, but it displays a high variance of residuals due in part to the uncertainty in the choice of forgetting and adaptive factors. Thomas presentes one of the better techniques for computing magnitude and phase in short times using the prony method [3]. This is inherently a least squares method which does not require synchronous sampling, and phase information can be obtained in about $1 / 2$ a cycle.

Unlike land-based power systems, ground faults on ship systems can cause serious damage in much less than one

\footnotetext{
${ }^{1}$ Both K. Davey and R. Hebner are with the Center for Electromechanics, University of Texas, 10100 Burnet Rd, EmE 133, Austin, TX 78758, (email k.davey@mail.utexas.edu, r.hebner@mail.utexas.edu).
}

cycle. The impedance of the ground path is at least two orders of magnitude smaller than those characterizing land systems. Damage suffered in recent ship board faults have solicited a request by the Navy for intelligent system healing response in $1 / 5^{\text {th }}$ of a cycle.

Advances in measurement techniques and primarily computing power have prompted research into new ways to detect faults. Jiao [4] proposes a combination of three phase negative sequences to construct a synthetic negative sequence vector which might be used to detect earth faults during power swings. During the fault, the negative sequence current is much smaller than the zero sequence component, and is therefore suggested as a guide indicator [5]. Styvaktakis recommends looking only at line voltage dip through a Kalman filter, but warns that events other than fault-induced dips would also trigger this indicator [6]. Qianli [7] suggests an index based on the maximum of the wavelet transformation of current with different scales. However, the fault phase is determined only by matching these characteristics to faults of different types. Wiot shares a view with these authors, i.e., that fault inceptions and switching events occurring in the vicinity of the protective relay can be detected by monitoring voltages and currents [8]. His method is based on a least squares minimization of the residual signal given by a short Fourier filter with two adapted coefficients.

The method proposed in this paper is believed to be more straightforward and involves directly computing the magnitude and phase of any signal based on three consecutive measurements. During a transient, either the frequency or phase can be treated as variable parameters. Shipboard systems have the demand that they must continue to function with the highest flexibility in the midst of a compromising situation. There is much argument in Navy circles for global grid control via a central processor because of the time required to manually reset triggered breakers under combat. Protection of tapped loads off trunk lines is necessary. This is to be accomplished by circuit breakers or in some cases differential relays. Of more interest in this paper is the control of switch settings regulating power flow down trunk lines from which multiple loads are tapped.

\section{MOTIVATION FOR THIS WORK}

Space does not permit examination of the reconfiguration issue in this paper. Nevertheless, reconfiguration is a primary motivation in this paper. Unlike a land based system, maintaining stability even during reconfiguration is considered a minor concern for shipboard systems because the generators are so tightly coupled. The emphasis rather is to deliver power 
to critical components when the grid has been severely compromised. The loads each maintain a priority weighting, and the goal is to maximize power delivery by priority weighting without violating trunk line current ratings. Lines feeding weapons are typically large, but have a small duty cycle. The objective is to operate the reconfiguration algorithm continuously, not just under combat, and make use of all underutilized lines. This objective becomes possible with the following two provisos:

1. The grid does not change shape, i.e., the equations defining the system are not dynamic, only the grid loads change.

2. The loads are quite dynamic, and constitute the drivers for the reconfiguration, one that can be updated in less than one cycle.

3. The trunk line switches have the capability of serving the dual purpose of providing fault protection on at the trunk line level, as well as reconfiguration capability.

With this motivation as background, important questions surface. Among those are "How can phasor information be computed in sub-cycle times?", "How can this information be used to represent a complex grid as a simpler equivalent system?", and "Does this model adequately represent dynamics during a fault transient?”.

\section{MODELING GUIDELINES}

The following theses are posited for a self-contained power system such as that on a shipboard system:

1. A near real time data stream of voltage and current can be translated in about one-fifth of a cycle to magnitude and phase information.

2. Magnitude and phase information allows trunk lines to be replaced by their equivalent series and parallel impedance.

3. Load control and power optimization can be implemented based on equivalent circuit representation.

4. Trunk line faults are earmarked by equivalent parallel impedances that drop below a threshold value. Fault control is realized by isolating both ends of the trunk line.

5. Resource allocation and smart distribution is realized by considering the grid an integrated system, and power can be delivered to the loads over multiple paths. Reconfiguration algorithms suitable for this type of system are beyond the scope of this paper, but are planned for a subsequent publication.

\section{REAL TIME TO PHASOR CONVERSION}

The sampling of current and voltage in time periods much less than the fundamental period is not useful by itself. In a time harmonic system with primary frequency $\omega$, these key parameters such as current and voltage can be represented over a short period of time with a magnitude and a phase. The ratio of the magnitudes and the difference of the phases is the key to rapid detection of faults. The currents on two separate lines 1 and 2 can be written as

$$
\begin{aligned}
& I_{1}=\alpha \cos (\omega t) \\
& I_{2}=\beta \cos (\omega t-\phi)
\end{aligned}
$$

One key to assessing system level status, including rapid fault detection, is the time history of the magnitude ratio $\alpha / \beta$ and the phase difference $\phi$. Note that during a fault or system transient, the magnitude and phase will change. The magnitude itself can be expressed as a sinusoid or a sum of sinusoids with frequencies different from $\omega$. The product of two sinusoids with different frequencies is equivalent to another sinusoid with a different frequency. Thus, this approach will handle the fact that the frequency is not really constant during a transient. The reader should note that $\mathrm{d} \phi / \mathrm{dt}$ represents the change in frequency.

Suppose the currents are being sampled with Hall effect probes at time interval $\delta t$. Define the signals $\mathrm{s}_{1}$ through $\mathrm{s}_{6}$ as

$$
\begin{aligned}
& s_{1}=A \cos (\omega t) \\
& s_{2}=A \cos (\omega(t+\delta t)) \\
& s_{3}=A \cos (\omega(t+2 \delta t)) \\
& s_{4}=B \cos (\omega t-\phi) \\
& s_{5}=B \cos (\omega(t+\delta t)-\phi) \\
& s_{6}=B \cos (\omega(t+2 \delta t)-\phi)
\end{aligned}
$$

Observe that

$$
\frac{A}{B}=\frac{s_{1} \cos (\phi) s_{2} \cos (\omega \delta t-\phi)}{s_{4}+s_{5} \cos (\omega \delta t)}
$$

The assumption of phase 0 for signal $s_{1}$ is not a limitation since $t$ is an arbitrary number. This expression is interesting but not very useful unless the phase is known a priori. The problem is that the time is unknown and the sampling period is short. Consider rearranging this into three equations,

$$
\begin{aligned}
s_{1} \cos (\omega t-\phi) & =\frac{A}{B} s_{4} \cos (\omega t) \\
s_{2} \cos (\omega(t+\delta t)-\phi) & =\frac{A}{B} s_{5}(\cos (\omega(t+\delta t))) \\
s_{3} \cos (\omega(t+2 \delta t)-\phi) & =\frac{A}{B} s_{6}(\cos (\omega(t+2 \delta t)))
\end{aligned}
$$

These constitute three equations and three unknowns, time t, which is of no concern, the phase $\phi$, and the magnitude ratio $\mathrm{A} / \mathrm{B}$. These equations can be solved analytically; that solution is quite long and is listed in the appendix. 


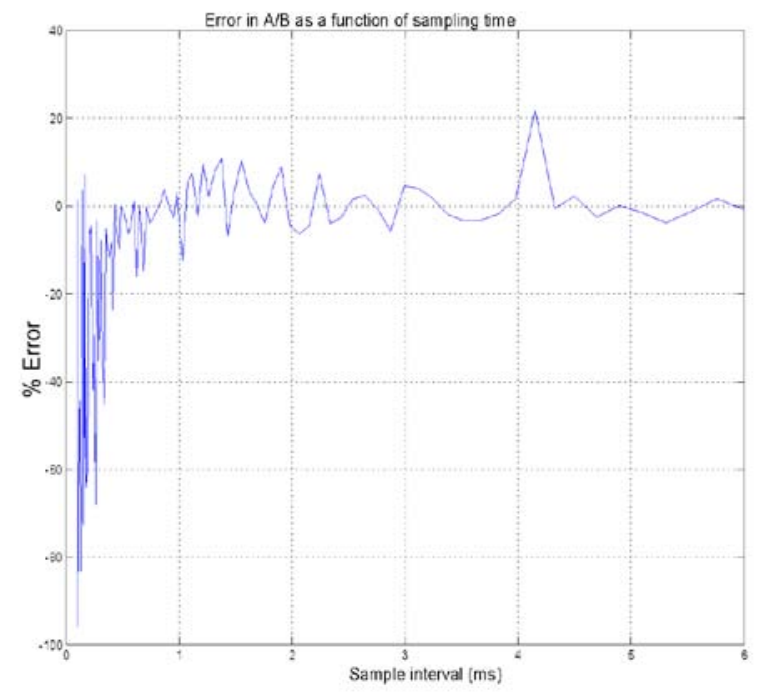

Fig. 1 Percent error as a function of sample time with a 5\% noise signal added to each reading.

Shown in Fig. 1 is the predicted percent error in the prediction of $\mathrm{A} / \mathrm{B}$ with only three sample points separated in time by the number of milliseconds marked on the abscissa. A 5\% random noise signal was added to the measurement of each signal, $s_{1}$ through $s_{6}$. In this test, the peak errors can never be expected to be lower than twice the noise signal. As shown below, this can be achieved if the signal is over sampled, i.e., a minimum of 4 data points.

When the measurement noise is reduced to $2 \%$, the error in the prediction changes to that shown in Fig. 2. These plots suggest that fault detection might conceivably be done reliably in 2-3 $\mathrm{ms}$, allowing a $\delta t$ of about $1 \mathrm{~ms}$.

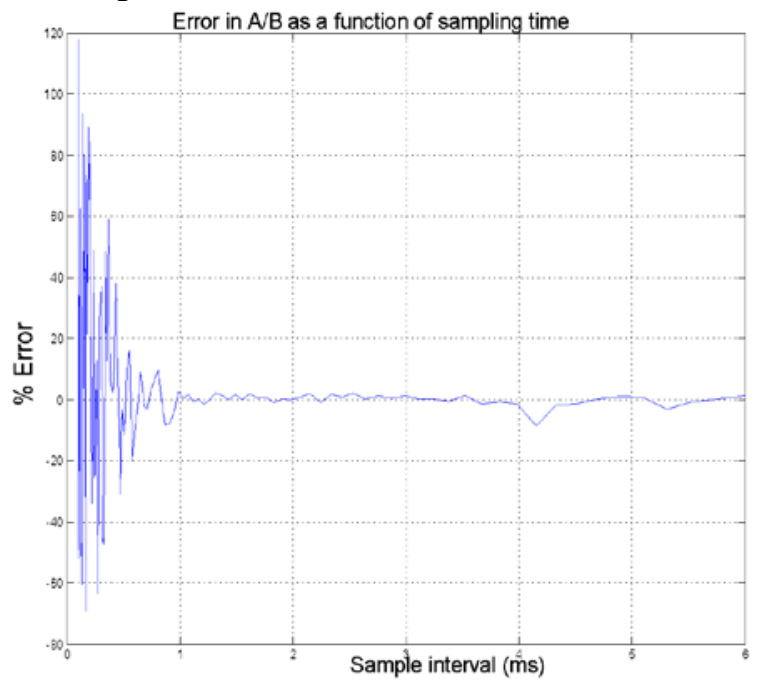

Fig. 2 Percent error when $2 \%$ noise is added to each sample reading.

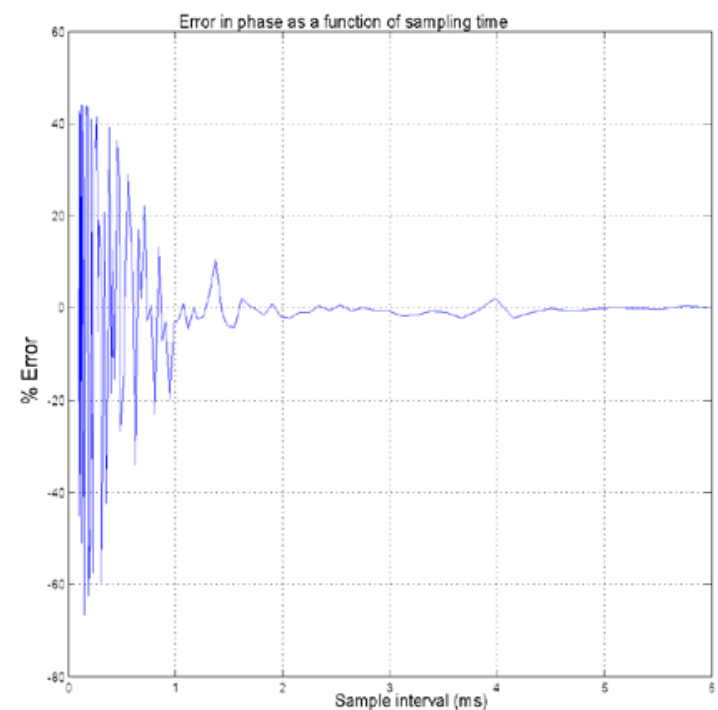

Fig. 3 Phase error when 5\% random noise is added to the measured signals.

Phase is not quite as sensitive to noise. Fig. 3 shows the phase prediction with a $5 \%$ random noise signal added to the measured signals. The results support the supposition that sampling on a small portion of a waveform allows an accurate assessment of the system status.

\section{EQUivalent CiRCUIT REPRESENTATION AND FAULt CONTROL}

Any power circuit grid can be laid out using trunk lines and loads on these lines as depicted in Fig. 4. Each trunk line has isolation switches on both ends, and sensors measuring voltage and current leaving their respective nodes. Multiple loads are hung off a trunk line. Although the load is variable, this load can be represented generally by a series impedance, $Z_{\mathrm{s}}$, on both sides of a parallel impedance, $Z_{p}$. The choice of an equal impedance on both sides of the parallel load is an arbitrary choice, but it will not affect load flow calculations.

These impedances are determined from the measurements for voltage and current. Using the results of the previous section and noting that the voltages and current are to be understood as phasor quantities, the impedances for the 5-9 trunk line annotated in the figure inset would follow as

$$
\begin{aligned}
& Z_{s}=\frac{2\left(V_{5}-V_{9}\right)}{\left(I_{5}-I_{9}\right)} \\
& Z_{p}=\frac{\left(V_{9} I_{5}-V_{5} I_{9}\right)}{I_{5}{ }^{2}-I_{9}{ }^{2}} .
\end{aligned}
$$

The procedure outlined in (5) is a technique for determining the relative magnitude and phase between any two signals. Of interest is the magnitude and phase of $Z_{s}$ and $Z_{p}$ between any two nodes i and j. Equation (5) must be repeated three times to get $Z_{s}$ since the general formula

$$
Z_{s i j}=2 \frac{V_{i}}{I_{i}} \frac{\left(1-\frac{V_{j}}{V_{i}}\right)}{\left(1-\frac{I_{j}}{I_{i}}\right)}
$$


depends on the relative magnitude and phase between $\mathrm{V}_{\mathrm{i}}$ and $I_{i}, V_{j}$ and $V_{i}$, and $I_{j}$ and $I_{i}$. A fourth magnitude ratio and phase is required to get $\mathrm{Zp}$, that between $\mathrm{V}_{\mathrm{j}}$ and $\mathrm{I}_{\mathrm{i}}$,

$Z_{p i j}=\frac{\left(\frac{V_{j}}{I_{i}}-\frac{V_{i}}{I_{i}} \cdot \frac{I_{j}}{I_{i}}\right)}{\left(1-\left(\frac{I_{j}}{I_{i}}\right)^{2}\right)}$

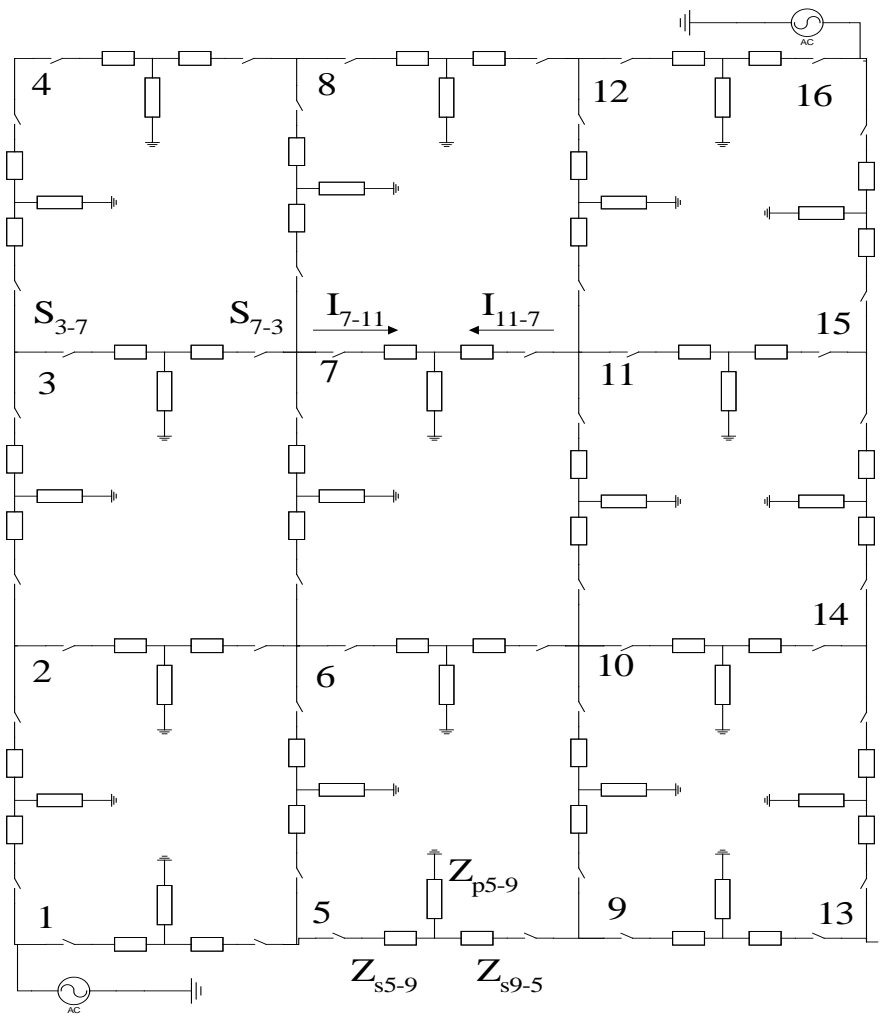

Fig. 4 Sixteen point grid with every trunk line represented by two switches, two series impedances, and one parallel load impedance.

Because (8) and (9) are updated in real time, these quantities are dynamic, not static. These two impedances adequately represent even the most nonlinear system for short time periods, and they are the key to fault control. Every trunk line has its own current rating according to the conductor size.
Assume a nominal operating system voltage $V_{n}$. The switches on both ends of a trunk line should be opened whenever

$$
\frac{V_{n}}{\frac{Z_{s}}{2}+Z_{p}} \geq I_{\text {trunk rating }}
$$

Consider what happens in the proposed system if a line to ground fault occurs somewhere in trunk line 2. The inrush current in trunk lines 1 and 3 is considerable, and all neighboring lines will register a voltage drop. Note that the key parameter for computing $Z_{p}$ in (7) and (9) is the difference between currents entering and leaving a given trunk line. When a fault occurs on trunk line 2, the large inrush current on the neighboring lines is a pass-through current. By focusing on $Z_{P}$, as opposed to current for fault control, the proposed approach avoids false trips of neighboring lines.

\section{A. Testing Fault Control}

\section{1) Passive Components}

Consider the expanded trunk line section shown in Fig. 5. The transmission line components, the balanced 3 phase parallel load, and the unbalanced three phase loads are represented as one trunk line section. There are three transmission section lines modeled as pi sections, 0.1 to $0.4 \mathrm{~km}$ long. Although the inductance, resistance, and capacitance of a real transmission line are distributed along the line, it can be modeled with reasonable accuracy as distributed $\mathrm{R}-\mathrm{L}$ segments sandwiched between multiple capacitors to ground. Two generators excite this coupled system at $60 \mathrm{~Hz}$. A $10 \mathrm{~m} \Omega$ line to ground fault occurs on phase A. The transient is simulated using a Runge Kutta algorithm using Matlab’s Simulink $\subset$. The voltage and current are measured every 0.5 ms. The upper two insets of Fig. 6 show the voltage and current predicted during this event. The lower two insets show the impedances $Z_{s}$ and $Z_{p}$ predicted through the evaluation of (8) and (9). Inductance L $\mathrm{di} / \mathrm{dt}$ voltages cause the initial transient jump in voltage witnessed. This simulation would suggest that about $3 \mathrm{~ms}$ will be required to properly diagnosis the fault. This number will be dictated by the $\mathrm{L} / \mathrm{R}$ ratio of components on the system. 


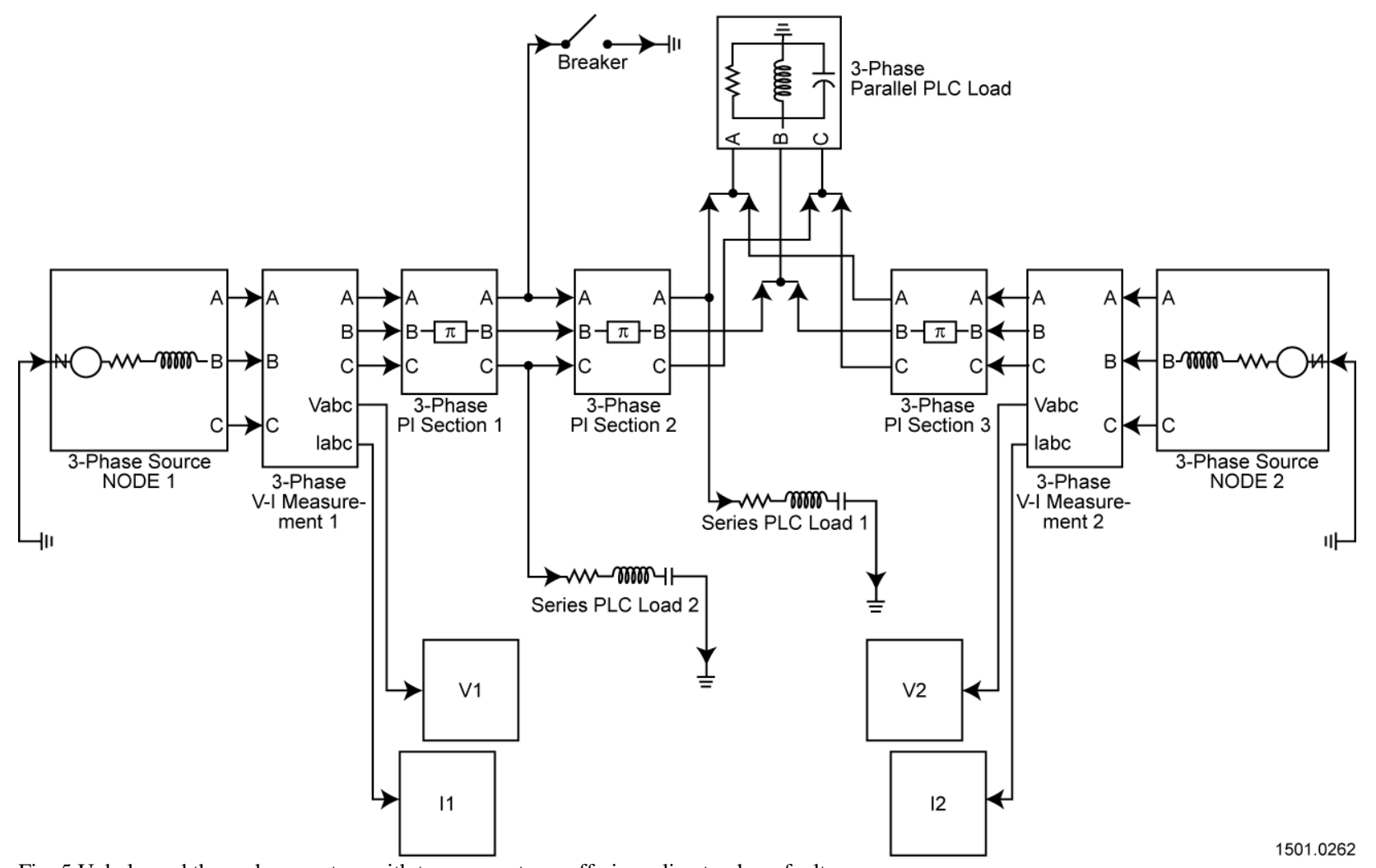

Fig. 5 Unbalanced three phase system with two generators suffering a line to phase fault.

Consider adding a $20 \mathrm{~kW}$ wound rotor induction motor with a
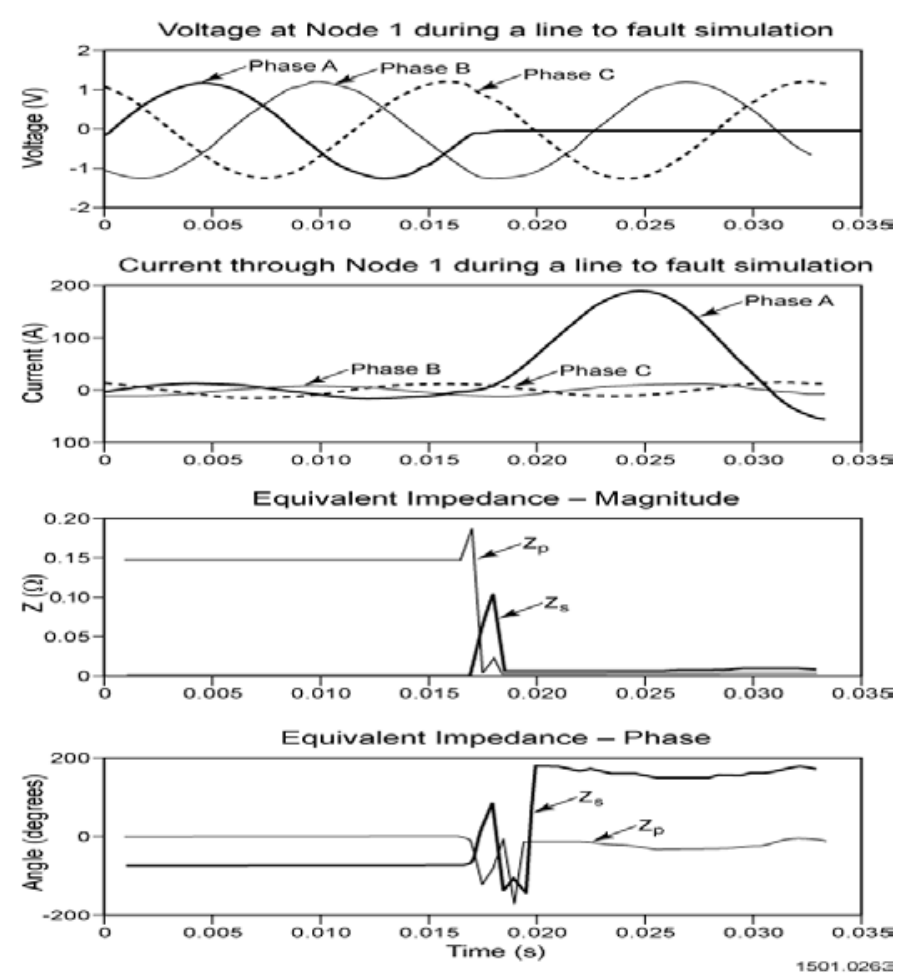

Fig. 6 Voltage, current, and impedance during a line to ground fault.

\section{2) Active Components}

$2 \mathrm{mH}$ magnetizing reactance to the model as suggested in Fig. 7. Of interest is the system performance if one phase of the motor is faulted to ground. Because of inertia, the rotor will continue to rotate through the important stages of the transient, introducing energy back into the grid. Because the fault is simulated 5 cycles into the start up cycle, the system is technically not quite in steady state equilibrium. The upper two insets of Fig. 8 show the voltage and current at node 2 simulated through the start-up transient. The lower two insets register $Z_{p}$ as calculated using (9). As witnessed by the previous analyses, these induction loads make the system perceive an initial increase in equivalent impedance $Z_{p}$ followed by a precipitous drop. The additional nuance added by the induction motor is the durative aspect of the fluctuation in $Z_{p}$. The motor causes sizeable variations in $Z_{p}$ through the first cycle after the fault, especially in phase. Note the considerable increase in equivalent $\mathrm{Z}_{\mathrm{s}}$ during the fault. 


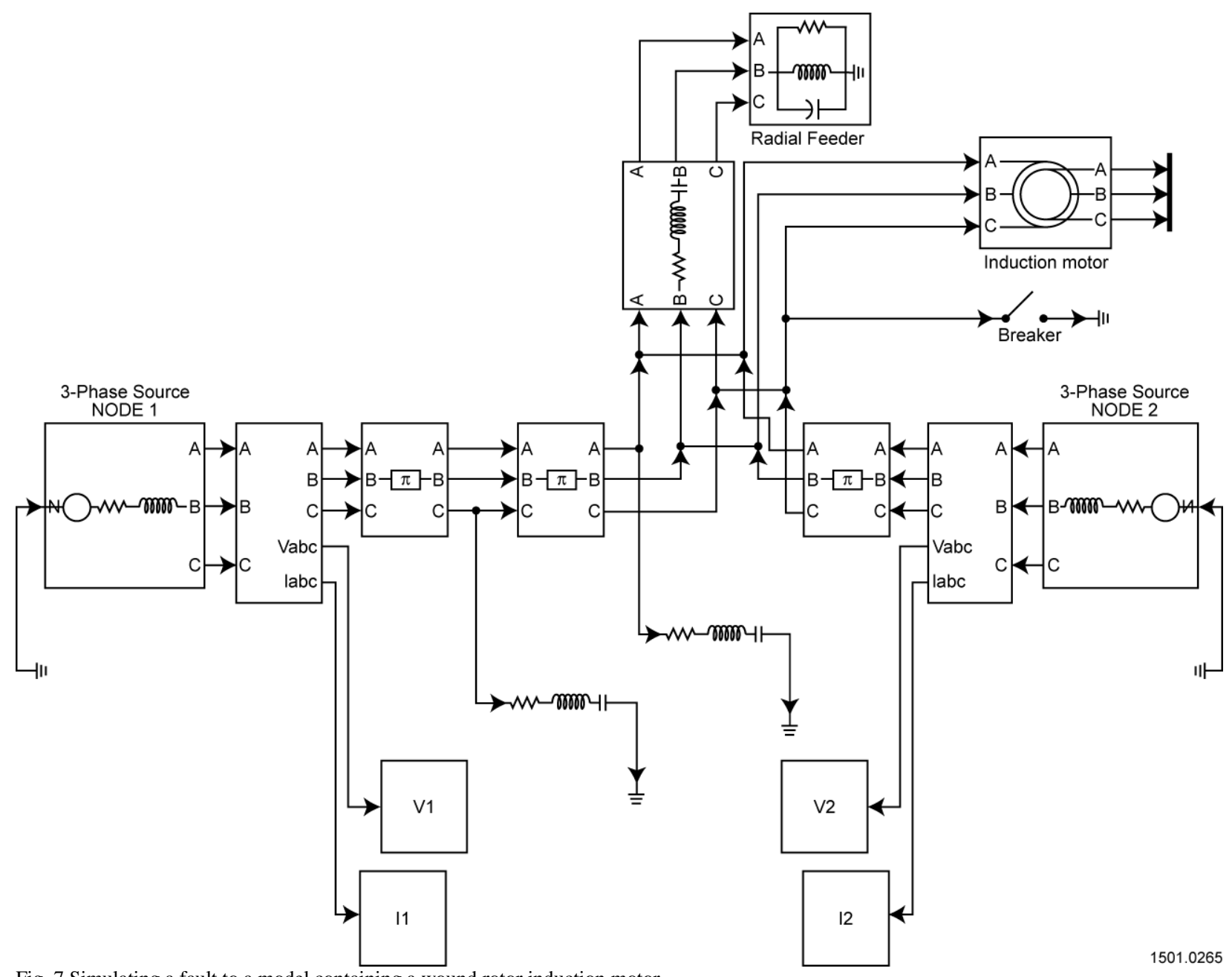

Fig. 7 Simulating a fault to a model containing a wound rotor induction motor. 

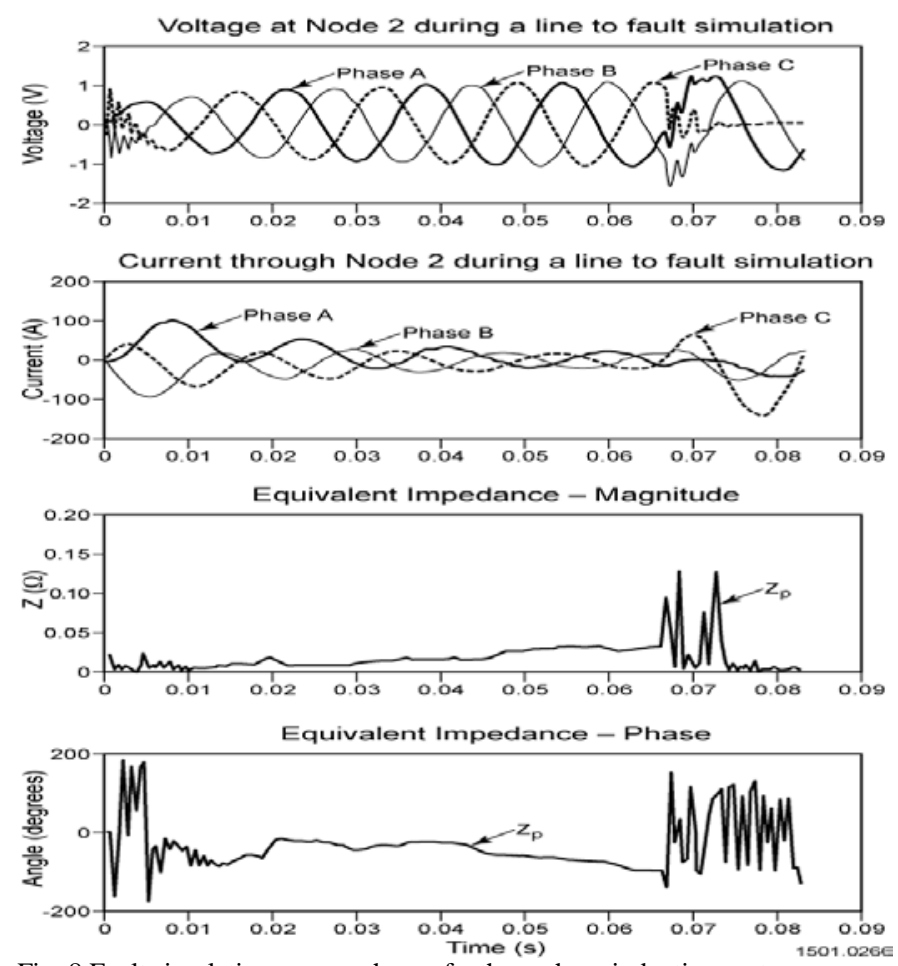

Fig. 8 Fault simulation on one phase of a three phase induction motor.

\section{DISCUSSION}

There are at least concerns that might be raised regarding the proposed system.

1. How will the proposed fault detection scheme distinguish between faults in loads tapped off trunk lines that should be cleared by protection dedicated to the load, and faults on the trunk lines themselves?

The proposed system has a multilayered protection scheme, similar to all land- based systems. Line inductance solves the problem on land systems. Circuit breakers on individual loads are triggered by current magnitude. Breakers and differential relays have sub-cycle response times. The transformer breaker is unlikely to trip from a home fault. Ship lines have low impedance, but they are surrounded by steel, which increases the inductance per foot. This inductance should allow the load fault protection to trip first.
2. How will the proposed fault detection technique discriminate between non-fault transients such as cold load pickup or transformer magnetizing inrush that are tapped off trunk lines?

Attention is warranted in this area. These cold loads are known, and their value must dictate the decision rule base for faults on trunk lines.

3. How will the high speed phasor estimation technique be of any use to a reconfiguration algorithm since it will capture transient overloads that should be ignored in a system configuration plan?

The working assumption inherent to this objection is that the reconfiguration is not running continuously. As computer speed and algorithm efficiency increase, this assumption is unwarranted, and reconfiguring the system through a transient might not only be possible, but advantageous. Stability on these tightly coupled systems is unlikely to be affected. The obvious method of dealing with the objection if the reconfiguration requires multiple cycles is to place a delay in the system to overcome the transient.

\section{CONCLUSIONS}

In the distribution strategy outlined, the grid becomes a cohesive composite with every trunk line having the capability of sharing part of the system load. It is no longer comprised of multiple isolated circuits. At the heart of the control strategy is a continuous monitoring of voltage and current at every trunk line. The salient points are as follows:

1. Treat the system as a number of feeder trunk lines, each with its own equivalent ' $\mathrm{T}$ ' impedance.

2. Continuously monitor current and voltage on either end of a trunk line.

3. Compute the relative magnitude and phase of these quantities using the formulae in Appendix A.

4. Register a fault through the magnitude change of the equivalent impedance $Z_{p}$

5. Determine possible locations of a fault on any given trunk line through the phase of $\mathrm{Z}_{\mathrm{p}}$ and a priori knowledge of the load.

Use a reconfiguration algorithm to maximize power delivery throughout the grid, subject to current rating constraints. The system equations are fixed, but the loads are dynamic. 
VIII. ApPendiX A - Magnitude And Phase Equations

$$
\frac{A}{B}=\frac{1}{(\text { term } a) \cdot(\text { term } b) \cdot \operatorname{term} c}
$$

\begin{tabular}{|c|c|}
\hline term $a$ & $\begin{array}{l}-2 \cdot s_{2}{ }^{2} \cdot \tan (\omega \cdot \delta t)^{2} \cdot s_{6} \cdot s_{4} \cdot s_{3} \cdot s_{1}+s_{2}^{2} \cdot \tan (\omega \cdot \delta t)^{2} \cdot s_{4}{ }^{2} \cdot s_{3}{ }^{2}+s_{2}{ }^{2} \\
\tan (\omega * \delta t)^{2} \cdot s_{6}{ }^{2} \cdot s_{1}{ }^{2}+s_{2}{ }^{2} \cdot s_{4}{ }^{2} \cdot s_{3}{ }^{2}+2 \cdot s_{2}{ }^{2} \cdot s_{6} \cdot s_{4} \cdot s_{3} \cdot s_{1}+s_{1}{ }^{2} \cdot s_{6}{ }^{2} \\
s_{2}{ }^{2}-4 \cdot s_{1}{ }^{2} \cdot s_{3} \cdot s_{5} \cdot s_{6} \cdot s_{2}+4 \cdot s_{3}{ }^{2} \cdot s_{1}{ }^{2} \cdot s_{5}{ }^{2}-4 \cdot s_{1} \cdot s_{3}{ }^{2} \cdot s_{2} \cdot s_{5} \cdot s_{4}\end{array}$ \\
\hline term $b$ & $\begin{array}{l}s_{4}^{2} \cdot s_{3}^{2} \cdot \tan (\omega \cdot \delta t)^{2} \cdot s_{5}^{2}+s_{3}^{2} \cdot s_{5}^{2} \cdot s_{4}^{2}-4 \cdot s_{4}^{2} \cdot s_{2} \cdot s_{5} \cdot s_{3} \cdot s_{6}+4 \\
s_{4}^{2} \cdot s_{6}^{2} \cdot s_{2}^{2}-4 \cdot s_{2} \cdot s_{5} \cdot s_{6}^{2} \cdot s_{4} \cdot s_{1}+s_{2} \cdot s_{5}^{2} \cdot s_{6} \cdot s_{3} \cdot s_{4} \cdot s_{1}-2 \cdot s_{4} \cdot s_{3} \\
s_{5}^{2} \cdot s_{6} \cdot \tan (\omega \cdot \delta t)^{2} \cdot s_{1}+\tan (\omega \cdot \delta t)^{2} \cdot s_{5}^{2} \cdot s_{6}{ }^{2} \cdot s_{1}{ }^{2} \cdot s_{5}^{2} \cdot s_{6}{ }^{2} \cdot s_{1}^{2}\end{array}$ \\
\hline term c & $\begin{array}{l}-2 \cdot s_{2}^{2} \cdot \tan (\omega \cdot \delta t)^{2} \cdot s_{6} \cdot s_{4} \cdot s_{3} \cdot s_{1}+s_{2}^{2} \cdot \tan (\omega \cdot \delta t)^{2} \cdot s_{4}^{2} \cdot s_{3}^{2}+s_{2}^{2} \\
\tan (\omega \cdot \delta t)^{2} \cdot s_{6}^{2} \cdot s_{1}^{2}+s_{2}^{2} \cdot s_{4}^{2} \cdot s_{3}^{2}+2 \cdot s_{2}^{2} \cdot s_{6} \cdot s_{3} \cdot s_{3} \cdot s_{1}+s_{1}^{2} \cdot s_{6}{ }^{2} \\
s_{2}{ }^{2}-4 \cdot s_{1}^{2} \cdot s_{3} \cdot s_{5} \cdot s_{6} \cdot s_{2}+4 \cdot s_{3}^{2} \cdot s_{1}^{2} \cdot s_{5}^{2}-4 \cdot s_{1} \cdot s_{3}^{2} \cdot s_{2} \cdot s_{5} \cdot s_{4}\end{array}$ \\
\hline & $\phi=\operatorname{atan}\left(\frac{-2}{((\text { term } d) \cdot(\text { term } e))^{(1 / 2)} \cdot \operatorname{term} f}\right),\left(\frac{1}{((\text { term } g) \cdot(\text { term } h))^{(1 / 2)} \cdot \operatorname{term} j}\right)$ \\
\hline term d & $\begin{array}{l}-2 \cdot s_{2}^{2} \cdot \tan (\omega \cdot \delta t)^{2} \cdot s_{6} \cdot s_{4} \cdot s_{3} \cdot s_{1}+s_{2}{ }^{2} \cdot \tan (\omega \cdot \delta t)^{2} \cdot s_{4}^{2} \cdot s_{3}^{2}+s_{2}{ }^{2} \cdot \tan (\omega \cdot \delta t)^{2} \\
s_{6} \cdot s_{1}^{2}+s_{2}^{2} \cdot s_{4}{ }^{2} \cdot s_{3}^{2}+2 \cdot s_{2}{ }^{2} \cdot s_{6} \cdot s_{4} \cdot s_{3} \cdot s_{1}+s_{1} \cdot s_{6}{ }^{2} \cdot s_{2}{ }^{2}-4 \cdot s_{1}{ }^{2} \cdot s_{3} \cdot s_{5} \cdot s_{6} \\
s_{2}+4 \cdot s_{3}{ }^{2} \cdot s_{1}{ }^{2} \cdot s_{5}{ }^{2}-4 \cdot s_{1} \cdot s_{3}{ }^{2} \cdot s_{2} \cdot s_{5} \cdot s_{4}\end{array}$ \\
\hline term e & $\begin{array}{l}s_{4}{ }^{2} \cdot s_{3}{ }^{2} \cdot \tan (\omega \cdot \delta t)^{2} \cdot s_{5}{ }^{2}+s_{3}{ }^{2} \cdot s_{5}{ }^{2} \cdot s_{4}{ }^{2}-4 \cdot s_{4}{ }^{2} \cdot s_{2} \cdot s_{5} \cdot s_{3} \cdot s_{6}+4 \cdot s_{4}{ }^{2} \cdot \\
s_{6}{ }^{2} \cdot s_{2}{ }^{2}-4 \cdot s_{2} \cdot s_{5} \cdot s_{6}{ }^{2} \cdot s_{4} \cdot s_{1}{ }^{2} \cdot s_{5}{ }^{2} \cdot s_{6} \cdot s_{3} \cdot s_{4} \cdot s_{1}-2 \cdot s_{4} \cdot s_{3} \cdot s_{5}{ }^{2} \cdot s_{6} \\
\tan (\omega \cdot \delta t)^{2} \cdot s_{1}+\tan (\omega \cdot \delta t)^{2} \cdot s_{5}{ }^{2} \cdot s_{6}{ }^{2} \cdot s_{1}{ }^{2}+s_{5}{ }^{2} \cdot s_{6}{ }^{2} \cdot s_{2}{ }^{2}\end{array}$ \\
\hline $\operatorname{term} f$ & $\left(s_{4} \cdot s_{3}-s_{6} \cdot s_{1}\right) \cdot\left(s_{4}+s_{2}-s_{5} \cdot s_{1}\right) \cdot\left(-s_{2} \cdot s_{6}+s_{5} \cdot s_{3}\right) \cdot \tan (\omega \cdot \delta t)$ \\
\hline term $g$ & $\begin{array}{l}-2 \cdot s_{2}{ }^{2} \cdot \tan (\omega \cdot \delta t)^{2} \cdot s_{6} \cdot s_{4} \cdot s_{3} \cdot s_{1}+s_{2}{ }^{2} \cdot \tan (\omega \cdot \delta t)^{2} \cdot s_{4}{ }^{2} \cdot s_{3}{ }^{2}+s_{2}{ }^{2} \cdot \\
\tan (\omega \cdot \delta t)^{2} \cdot s_{6}{ }^{2} \cdot s_{1}{ }^{2}+s_{2}{ }^{2} \cdot s_{4}{ }^{2} \cdot s_{3}{ }^{2}+2 \cdot s_{2}{ }^{2} \cdot s_{6} \cdot s_{4} \cdot s_{3} \cdot s_{1}+s_{1}{ }^{2} \cdot s_{6}{ }^{2} \\
s_{2}{ }^{2}-4 \cdot s_{1}{ }^{2} \cdot s_{3} \cdot s_{5} \cdot s_{6} \cdot s_{2}+4 \cdot s_{3}{ }^{2} \cdot s_{1}{ }^{2} \cdot s_{5}{ }^{2}-4 \cdot s_{1} \cdot s_{3}{ }^{2} \cdot s_{2} \cdot s_{5} \cdot s_{4}\end{array}$ \\
\hline term $h$ & $\begin{array}{l}s_{4}^{2} \cdot s_{3}^{2} \cdot \tan (\omega \cdot \delta t)^{2} \cdot s_{5}^{2}+s_{3}^{2} \cdot s_{5}^{2} \cdot s_{4}^{2}-4 \cdot s_{4}^{2} \cdot s_{2} \cdot s_{5} \cdot s_{3} \cdot s_{6}+4 \\
s_{4}{ }^{2} \cdot s_{6}{ }^{2} \cdot s_{2}{ }^{2}-4 \cdot s_{2} \cdot s_{5} \cdot s_{6}{ }^{2} \cdot s_{4} \cdot s_{1}+2 \cdot s_{5}{ }^{2} \cdot s_{6} \cdot s_{3} \cdot s_{4} \cdot s_{1}-2 \cdot s_{4} \cdot s_{3} \\
s_{5}{ }^{2} \cdot s_{6} \cdot \tan (\omega \cdot \delta t)^{2} \cdot s_{1}+\tan (\omega \cdot \delta t)^{2} \cdot s_{5}{ }^{2} \cdot s_{6}{ }^{2} \cdot s_{1}{ }^{2} \cdot s_{5}{ }^{2} \cdot s_{6}{ }^{2} \cdot s_{1}{ }^{2}\end{array}$ \\
\hline term $j$ & $\begin{array}{l}-6 \cdot s_{2} \cdot s_{6} \cdot s_{5} \cdot s_{1} \cdot s_{3} \cdot s_{4}-s_{1}{ }^{2} \cdot s_{6}{ }^{2} \cdot s_{2} \cdot s_{5}{ }^{2}+2 \cdot s_{2}{ }^{2} \cdot s_{6} \cdot s_{4}{ }^{2} \cdot s_{3}+2 \cdot \\
s_{2}{ }^{2} \cdot s_{6}{ }^{2} \cdot s_{4} \cdot s_{1}+2 \cdot s_{6} \cdot s_{3} \cdot s_{1}{ }^{2} \cdot s_{5}{ }^{2}+2 \cdot s_{5}{ }^{2} \cdot s_{3}{ }^{2} \cdot s_{1} \cdot s_{4}-s_{5} \cdot s_{3}{ }^{2} \cdot s_{4}{ }^{2} \cdot \\
s_{2}+s_{3}{ }^{2} \cdot \tan (\omega \cdot \delta t)^{2} \cdot s_{5} \cdot s_{2} \cdot s_{4}{ }^{2}-2 \cdot s_{3} \cdot \tan (\omega \cdot \delta t)^{2} \cdot s_{5} \cdot s_{2} \cdot s_{4} \cdot s_{6} \cdot \\
s_{1}+\tan (\omega \cdot \delta t)^{2} \cdot s_{5} \cdot s_{2} \cdot s_{1}{ }^{2} \cdot s_{6}{ }^{2}\end{array}$ \\
\hline
\end{tabular}




\section{REFERENCES}

[1] A.K. Pradham, A. Routray, and D. Sethi, "Voltage phasor estimation using complex linear complex filter," 8th IEE Conference on Developments in Power System Protection, Vol. 1, April 2004, pp. 24-27.

[2] I. Kamwa and R.Grondin, "Fast adaptive schemes for tracking voltage phasor and local frequency in power transmission and distribution systems," IEEE Transactions on Power Delivery, Vol. 7, No. 2, April 1992, pp. 789-795.

[3] D. W. P. Thomas and M. S. Woolfson, "Voltage and current phasor estimation during abnormal conditions for transmission line protection schemes," 6th IEE Conference on Power System Protection, March 25-27 1997, pp. 266-269.

[4] S. Jiao, Z. Bo, W. Liu, and Q. Yang, "New Principles to detect faults during Power Swings,” Developments in Power System Protection Conference, IEE Publication No. 479, 2001, pp. 515-518.

[5] Z. Xiangjun, K. Li, W. Chan, and Y. Xianggen, "Novel Techniques for Earth Fault Feeder Detection Based on Negative Sequence Current in Industry Power Systems,” Industry Applications Conference, 2001.Thirty-Sixth IAS Annual Meeting. Conference Record of the 2001 IEEE , Volume: 3 , 30 Sept.4 Oct. 2001, pp.1831 - 1837.

[6] E. Styvaktakis, I. Gu, and M. Bollen, "Voltage dip detection and power system transients," IEEE Power Engineering Society Summer Meeting, Volume: 1 , 15-19 July 2001, pp. 683 - 688.

[7] S. Qianli, D. Xinzhou, Z. Bo, and F. Jiang, "New approach of fault detection and fault phase selection based on initial current traveling waves," IEEE Power Engineering Society Summer Meeting, Volume: 1 , 21-25 July 2002, pp. $393-397$.

[8] D. Wiot, "A new adaptive transient monitoring scheme for detection of power system faults," IEEE Trans. on Power System Delivery, vol. 19, no. 1, Jan. 2004, pp. 42-48. 